\title{
String Physics at the LHC
}

\author{
Luis A. Anchordoqui \\ Department of Physics, University of Wisconsin-Milwaukee, Milwaukee, WI 53201, USA
}

\begin{abstract}
The LHC program will include the identification of events with single high- $k_{T}$ photons as probes of new physics. We show that this channel is uniquely suited to search for experimental evidence of TeV-scale open string theory.
\end{abstract}

Keywords: D-brane phenomenology, Regge recurrences

PACS: $11.25 . \mathrm{Wx}, 13.85 . \mathrm{Ni}, 13.85 . \mathrm{Qk}$

The saga of the standard model (SM) is still exhilarating because it leaves all questions of consequence unanswered. The most evident of these questions concerns quantum gravity. In particular, renormalization, the technique allowing finite predictions for processes involving the electroweak and strong forces, fails when gravity is taken into account. String theory is currently the only known consistent framework to overcome the problem of non-renormalizability and quantize gravity [1]. Within this framework, the notion of elementary point-particle is replaced by an extended object of vanishing width that has two different topologies, corresponding to open and closed strings 1 The fundamental particles thus far appear point-like to us because the experimental energies probed by colliders are too small to excite the string oscillation modes. In addition, the apparent weakness of gravity relative to the other fundamental forces can be understood in string theory as a consequence of the gravitational force "leaking" into unseen compact dimensions transverse to a braneworld where we are confined [2]. The distance at which quantum gravity comes into play could be then $\mathscr{O}\left(10^{-18} \mathrm{~m}\right)$. Showld nature be so cooperative, one would expect to see a few string states produced at the LHC.

In the perturbative regime, gauge interactions emerge as excitations of open strings with endpoints confined on D-branes [3]. The basic unit of gauge invariance for D-brane constructions is a $U(1)$ field, and so one can stack up $N$ identical D-branes to generate a $U(N)$ theory with the associated $U(N)$ gauge group. Gauge bosons arise from strings terminating on one stack of D-branes, whereas chiral matter fields are obtained from string stretching between two stacks [4]. Each of the two strings endpoints carries a fundamental charge with respect to the stack of branes on which it terminates. Mater fields

\footnotetext{
${ }^{1}$ The closed string sector automatically includes gravitation, because it contains an oscillation mode propagating in vacuum corresponding to a spin 2 particle which can be identified with the graviton.
}

thus posses quantum numbers associated with a bifundamental representation. None of them has three quantum numbers of the sort we are used to: $S U(3) \times S U(2) \times$ $U(1)_{Y}$; rather they all have two quantum numbers at the expense of introducing additional $U(1)$ 's into the theory [5, 6].

To develop our program in the simplest way, we will work within the construct of a minimal model in which we consider scattering processes which take place on the (color) $U(3)$ stack of D-branes. In the bosonic sector, the open strings terminating on this stack contain, in addition to the $S U(3)$ octet of gluons, an extra $U(1)$ boson $\left(C_{\mu}\right.$, in the notation of [6]), most simply the manifestation of a gauged baryon number symmetry. The $U(1)_{Y}$ boson $Y_{\mu}$, which gauges the usual electroweak hypercharge symmetry, is a linear combination of $C_{\mu}$, the $U(1)$ boson $B_{\mu}$ terminating on a separate $U(1)$ brane, and perhaps a third additional $U(1)$ (say $W_{\mu}$ ) sharing a $U(2)$ brane which is also a terminus for the $S U(2)_{L}$ electroweak gauge bosons $W_{\mu}^{a}$. Thus, critically for our purposes, the photon $A_{\mu}$, which is a linear combination of $Y_{\mu}$ and $W_{\mu}^{3}$ will participate with the gluon octet in (string) tree level scattering processes on the color brane, processes which in the SM occur only at one-loop level.

The process we consider (at the parton level) is $g g \rightarrow$ $g \gamma$, where $g$ is an $S U(3)$ gluon and $\gamma$ is the photon. As explicitly calculated below, this will occur at string disk (tree) level, and will be manifest at LHC as a non-SM contribution to $p p \rightarrow \gamma+$ jet. A very important property of string disk amplitudes is that they are completely model-independent; thus the results presented below are robust, because they hold for arbitrary compactifications of superstring theory from ten to four dimensions, including those that break supersymmetry.

In a helicity basis, scattering amplitudes are classified according to the number of $( \pm)$ states of the external partons. The Maximum Helicity Violating (MHV) amplitude describes the configuration with the highest difference of (+) and ( -$)$ states, e.g. $n-2$ for a $n$-gluon amplitude at tree level [7]. Assume that two vector bosons, 
with the momenta $k_{1}$ and $k_{2}$, in the $U(N)$ gauge group states corresponding to the generators $T^{a_{1}}$ and $T^{a_{2}}$ (here in the fundamental representation), carry negative helicities while the other two, with the momenta $k_{3}$ and $k_{4}$ and gauge group states $T^{a_{3}}$ and $T^{a_{4}}$, respectively, carry positive helicities. Then the partial amplitude for such an MHV configuration is given by [8, 9]

$$
\begin{aligned}
A\left(1^{-}, 2^{-}, 3^{+}, 4^{+}\right) & =4 g^{2} \operatorname{Tr}\left(T^{a_{1}} T^{a_{2}} T^{a_{3}} T^{a_{4}}\right) \\
& \times \frac{\langle 12\rangle^{4}}{\langle 12\rangle\langle 23\rangle\langle 34\rangle\langle 41\rangle} \\
& \times V\left(k_{1}, k_{2}, k_{3}, k_{4}\right),
\end{aligned}
$$

where $g$ is the $U(N)$ coupling constant, $\langle i j\rangle$ are the standard spinor products, and the Veneziano formfactor,

$$
V\left(k_{1}, k_{2}, k_{3}, k_{4}\right)=V(s, t, u)=\frac{\Gamma(1-s) \Gamma(1-u)}{\Gamma(1+t)},
$$

is the function of Mandelstam variables, here normalized in the string units:

$$
s=\frac{2 k_{1} k_{2}}{M_{s}^{2}}, t=\frac{2 k_{1} k_{3}}{M_{s}^{2}}, u=\frac{2 k_{1} k_{4}}{M_{s}^{2}},
$$

with $s+t+u=02$ In order to obtain the cross section for the (unpolarized) partonic subprocess $g g \rightarrow g \gamma$, we take the squared moduli of individual amplitudes, sum over final polarizations and colors, and average over initial polarizations and colors. The two most interesting energy regimes of $g g \rightarrow g \gamma$ scattering are far below the string mass scale $M_{s}$ and near the threshold for the production of massive string excitations. At low energies $(s, t, u \ll$ 1) [11]

$$
|\mathscr{M}(g g \rightarrow g \gamma)|^{2} \approx g^{4} Q^{2} C(N) \frac{\pi^{4}}{4 M_{s}^{8}}\left(\hat{s}^{4}+\hat{t}^{4}+\hat{u}^{4}\right),
$$

where $C(N)=\left[2\left(N^{2}-4\right)\right] /\left[N\left(N^{2}-1\right)\right]$. The absence of zero mass poles, at $s=0$ etc., translated into the terms of effective field theory, confirms that there are no exchanges of massless particles contributing to this process. On the other hand, near the string threshold $(s \approx 1)[11]$

$$
|\mathscr{M}(g g \rightarrow g \gamma)|^{2} \approx 4 g^{4} Q^{2} C(N) \frac{M_{s}^{8}+\hat{t}^{4}+\hat{u}^{4}}{M_{s}^{4}\left(\hat{s}-M_{s}^{2}\right)^{2}} .
$$

The singularity at $\hat{s}=M_{s}^{2}$ needs softening to a BreitWigner form, reflecting the finite decay widths of resonances propagating in the $s$ channel. Due to averaging over initial polarizations, Eq. (5) contains additively

\footnotetext{
2 We use the standard notation of [10], although the gauge group generators are normalized here in a different way, according to $\operatorname{Tr}\left(T^{a} T^{b}\right)=$ $\frac{1}{2} \delta^{a b}$.
}

contributions from both spin $J=0$ and $\operatorname{spin} J=2$ gluonic Regge recurrences, created by the incident gluons in the helicity configurations $( \pm \pm)$ and $( \pm \mp)$, respectively. The $M_{s}^{8}$ term in Eq. (5) originates from $J=0$, and the $\hat{t}^{4}+\hat{u}^{4}$ piece reflects $J=2$ activity. Since the resonance widths are spin-dependent, $\Gamma^{J=0} \approx 75\left(M_{S} / \mathrm{TeV}\right) \mathrm{GeV}$ and $\Gamma^{J=2} \approx 45\left(M_{S} / \mathrm{TeV}\right) \mathrm{GeV}$ [12], the pole term (5] should be smeared as

$$
\begin{aligned}
|\mathscr{M}(g g \rightarrow g \gamma)|^{2} & \simeq \frac{4 g^{4} Q^{2} C(N)}{M_{s}^{4}} \\
& \times\left[\frac{M_{s}^{8}}{\left(\hat{s}-M_{s}^{2}\right)^{2}+\left(\Gamma^{J=0} M_{s}\right)^{2}}\right. \\
& \left.+\frac{\hat{t}^{4}+\hat{u}^{4}}{\left(\hat{s}-M_{s}^{2}\right)^{2}+\left(\Gamma^{J=2} M_{s}\right)^{2}}\right] .
\end{aligned}
$$

In what follows we will take $N=3$, set $g$ equal to the QCD coupling constant $\left(g^{2} / 4 \pi=0.1\right)$. Before proceeding with numerical calculation, we need to make precise the value of $Q$. If we were considering the process $g g \rightarrow C g$, where $C$ is the U(1) gauge field tied to the $U(3)$ brane, then $Q=\sqrt{1 / 6}$ due to the normalization condition. However, for $g g \rightarrow \gamma g$ there are two additional projections: from $C_{\mu}$ to the hypercharge boson $Y_{\mu}$, giving a mixing factor $\kappa$; and from $Y_{\mu}$ onto a photon, providing an additional factor $\cos \theta_{W}\left(\theta_{W}=\right.$ Weinberg angle $)$. The $C-Y$ mixing coefficient is model dependent: in the minimal model [6] it is quite small, around $\kappa \simeq 0.12$ for couplings evaluated at the $Z$ mass, which is modestly enhanced to $\kappa \simeq 0.14$ as a result of $R G$ running of the couplings up to $2.5 \mathrm{TeV}$. It should be noted that in models [5] possessing an additional $U(1)$ which partners $S U(2)_{L}$ on a $U(2)$ brane, the various assignment of the charges can result in values of $\kappa$ which can differ considerably from 0.12 . In what follows, we take as a fiducial value $\kappa^{2}=0.02$. Thus, if (6) is to describe $g g \rightarrow \gamma g$,

$$
Q^{2}=\frac{1}{6} \kappa^{2} \cos ^{2} \theta_{W} \simeq 2.55 \times 10^{-3}\left(\kappa^{2} / 0.02\right) .
$$

One would hope that the resonance would be visible in data binned according to the invariant mass $M$ of the photon + jet, setting cuts on photon and jet rapidities, $y_{1}, y_{2}<y_{\max }$, respectively. With the definitions $Y \equiv \frac{1}{2}\left(y_{1}+y_{2}\right)$ and $y \equiv \frac{1}{2}\left(y_{1}-y_{2}\right)$, the cross section per interval of $M$ for $p p \rightarrow \gamma+$ jet $+X$ is given by [13]

$$
\begin{aligned}
\frac{d \sigma}{d M} & =M \tau \sum_{i j k}\left[\int_{-Y_{\max }}^{0} d Y f_{i}\left(x_{a}, M\right) f_{j}\left(x_{b}, M\right)\right. \\
& \times\left.\int_{-\left(y_{\max }+Y\right)}^{y_{\max }+Y} d y \frac{d \sigma}{d \hat{t}}\right|_{i j \rightarrow \gamma k} \frac{1}{\cosh ^{2} y} \\
& +\int_{0}^{Y_{\max }} d Y f_{i}\left(x_{a}, M\right) f_{j}\left(x_{b}, M\right) \\
& \left.\times\left.\int_{-\left(y_{\max }-Y\right)}^{y_{\max }-Y} d y \frac{d \sigma}{d \hat{t}}\right|_{i j \rightarrow \gamma k} \frac{1}{\cosh ^{2} y}\right],
\end{aligned}
$$




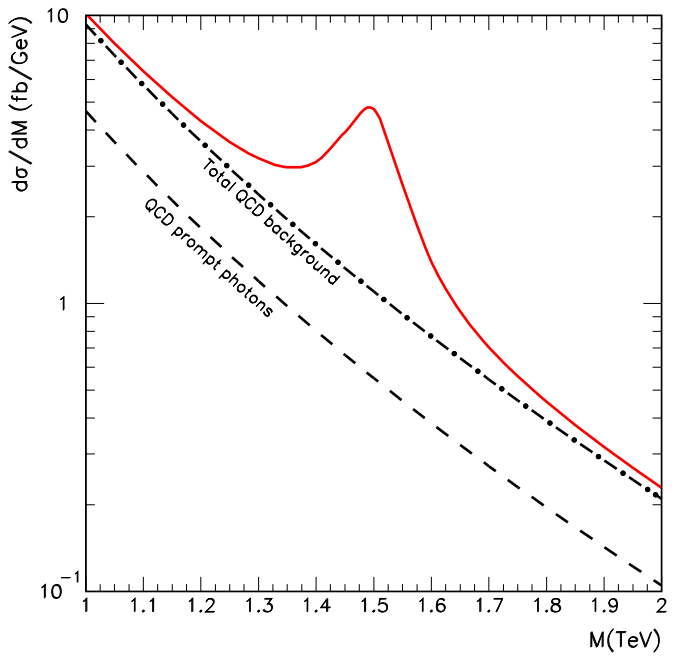

FIGURE 1. Invariant mass spectrum: $d \sigma / d M$ (units of $\mathrm{fb} / \mathrm{GeV}) v s . M(\mathrm{TeV})$ is plotted for the case of SM QCD background (dot-dashed line) and (first resonance) string signal + background (solid line). After minimization of misidentified $\pi^{0}$ 's from high- $p_{T}$ jets [14], the noise is increased by a factor of $\sqrt{2}$ over the direct photon QCD contribution (dashed line).

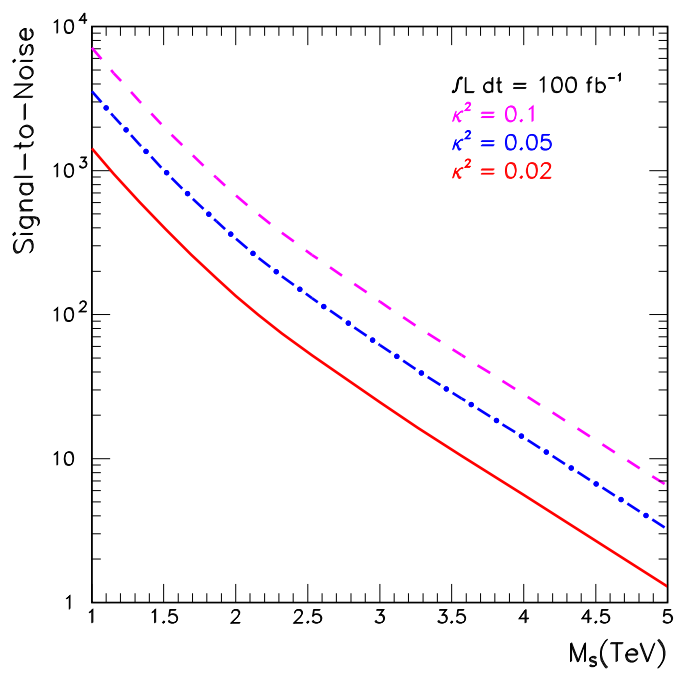

FIGURE 2. signal-to-noise ratio for an integrated luminosity of $100 \mathrm{fb}^{-1}$. The solid line is for $\kappa^{2}=0.02$, the dot-dahsed line is for $\kappa^{2}=0.05$, and the dashed line is for an optimistic case with $\kappa^{2}=0.1$.

where $i, j, k$ are different partons, $\tau=M^{2} / s, x_{a}=\sqrt{\tau} e^{Y}$, and $x_{b}=\sqrt{\tau} e^{-Y}$. The kinematics of the scattering provides the relation $k_{T}=M /(2 \cosh y)$, which when combined with the standard cut $k_{T}>k_{T \text {,min }}$, imposes a lower bound on $y$ to be implemented in the limits of integration. The $Y$ integration range in Eq. (8), $Y_{\max }=$ $\min \left\{\ln (1 / \sqrt{\tau}), y_{\max }\right\}$, comes from requiring $x_{a}, x_{b}<1$ together with the rapidity cuts $\left|y_{1}\right|,\left|y_{2}\right| \leq 2.4$. Finally, the Mandelstam invariants occurring in the cross section are given by $\hat{s}=M^{2}, \hat{t}=-\frac{1}{2} M^{2} e^{-y} / \cosh y$, and $\hat{u}=-\frac{1}{2} M^{2} e^{+y} / \cosh y$.

In Figure 1 we show a representative plot of this cross section, for $M_{s}=1.5 \mathrm{TeV}$. Standard bump-hunting methods, such as calculating cumulative cross sections, $\sigma\left(M_{0}\right)=\int_{M_{0}}^{\infty} \frac{d \sigma}{d M} d M$, and searching for regions with significant deviations from the QCD background, may allow to find an interval of $M$ suspected of containing a bump. With the establishment of such a region, one may calculate a signal-to-noise ratio, with the signal rate estimated in the invariant mass window $\left[M_{s}-2 \Gamma, M_{s}+2 \Gamma\right]$. The signal-to-noise ratio is shown in Figure 2] It is clearly seen that even for relatively small mixing, $100 \mathrm{fb}^{-1}$ of LHC data could probe deviations from SM physics associated with $\mathrm{TeV}$-scale strings at a $5 \sigma$ significance, for $M_{s} \lesssim 4 \mathrm{TeV}$

\section{ACKNOWLEDGMENTS}

This talk is based on several works done in collaboration with Haim Goldberg, Satoshi Nawata, and Tom Taylor. LAA is supported by the US NSF and the UWM RGI.

\section{REFERENCES}

1. J. Scherk and J. H. Schwarz, Nucl. Phys. B 81, 118 (1974).

2. I. Antoniadis, N. Arkani-Hamed, S. Dimopoulos and G. R. Dvali, Phys. Lett. B 436, 257 (1998) |arXiv:hep-ph/9804398|.

3. J. Polchinski, arXiv:hep-th/9611050

4. R. Blumenhagen, B. Kors, D. Lust and S. Stieberger, Phys. Rept. 445, 1 (2007) |arXiv:hep-th/0610327|.

5. I. Antoniadis, E. Kiritsis and T. N. Tomaras, Phys. Lett. B 486, 186 (2000). [arXiv:hep-ph/0004214].

6. D. Berenstein and S. Pinansky, Phys. Rev. D 75, 095009 (2007) [arXiv:hep-th/0610104].

7. S. J. Parke and T. R. Taylor, Phys. Rev. Lett. 56, 2459 (1986).

8. S. Stieberger and T. R. Taylor, Phys. Rev. Lett. 97, 211601 (2006) [arXiv:hep-th/0607184].

9. S. Stieberger and T. R. Taylor, Phys. Rev. D 74, 126007 (2006) [arXiv:hep-th/0609175].

10. M. L. Mangano and S. J. Parke, Phys. Rept. 200, 301 (1991) |arXiv:hep-th/0509223].

11. L. A. Anchordoqui, H. Goldberg, S. Nawata and T. R. Taylor, Phys. Rev. Lett. 100, 171603 (2008) arXiv:0712.0386 [hep-ph]].

12. L. A. Anchordoqui, H. Goldberg and T. R. Taylor, arXiv:0806.3420 [hep-ph].

13. L. A. Anchordoqui, H. Goldberg, S. Nawata and T. R. Taylor, Phys. Rev. D (to be published) |arXiv:0804.2013 [hep-ph]].

14. P. Gupta, B. C. Choudhary, S. Chatterji, S. Bhattacharya and R. K. Shivpuri, arXiv:0705.2740 [hep-ex]. 